\title{
Comparative study of change detection methods for multi-temporal fundus images.
}

\author{
Beulah David D ${ }^{1^{*}}$, Dorai Rangaswamy ${ }^{2}$ \\ ${ }^{1}$ Department of Computer Science and Engineering, Sathyabama University, Chennai, Tamil Nadu, India \\ ${ }^{2}$ Adi Shankara Institute of Engineering and Technology, Kalady, Kerala, India
}

\begin{abstract}
A procedure which has a tendency to find the micro aneurysms and hemorrhages in shading color fundus picture utilizing computerized or semi-mechanized divisions for vein division in fundus structure upheld by a discriminatively prepared, completely associated contingent irregular field demonstrate. Since it has high exactness it is prudent and checks by its size thus utilized as a part of early discovery, analysis and treatment. Since Image division assumes a basic part in numerous therapeutic applications. The objective of this research or to conquer the issue a restrictive irregular field demonstrates with a ton of open possibilities, exploiting ongoing outcomes sanctionative intelligent reasoning of completely associated models for all intents and purposes continuously. The proposed framework method which is a six stage process from spatial adjustment till classification is expected to improve the information pictures taken for activity. By following this sort of system early recognition of the variations from the norm made without the loss of the blood tests every last time that happens while checking for the irregularity. The outcomes show the solid execution of the proposed strategy in recognizing the micro aneurysms and hemorrhages in fundus pictures of various determination and quality and from various securing frameworks. Additionally work concentrating on splendid injury and neo vessel location will finish the proposed framework effectively than other existing methods.
\end{abstract}

Keywords: Fundus photography, Diabetic macular edema, Choroidal neovascularisation, Diabetic retinopathy. Accepted on September 14, 2018

\section{Introduction}

Catching the back of the eye fundus is named as fundus photography. Uncommon cameras appended with specific magnifying lens and glimmer empowered cameras are broadly gotten to. The types of gear, models and innovation of such photography give an extraordinary upset to the clinical world. A portion of the fundus camera makes are just accessible in the market [1].

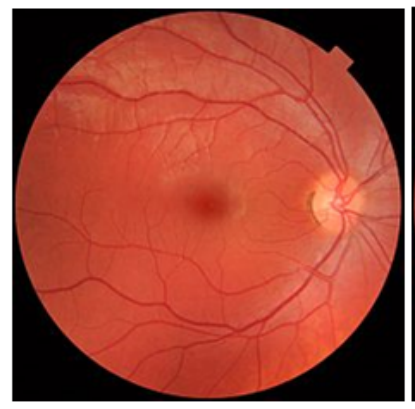

(a)

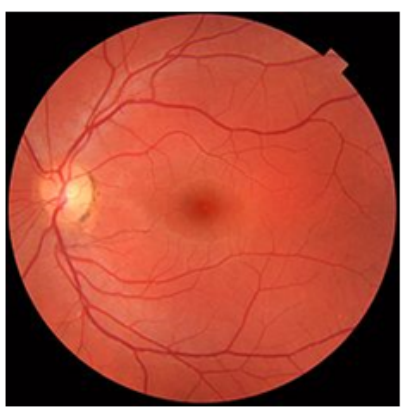

(b)
Figure 1. Fundus photography of (a) right eye (b) left eye.
Figure 1 demonstrates the ordinary fundus photography and the picture hinted at has no illness by any stretch of the imagination. The dark in the focal point of the picture is named as macula and the optic plate is the white hover appeared along the nerves. The optic disks (OD) of the picture indicate just some pigmentation in the sidelong side [1]. The orange shading in the given picture demonstrates the vitamin multifaceted nature in the retina. Hence by review the picture we can decide the age gathering of the individual like the left picture of Figure 1 since lighter zones in the picture demonstrates that the photography is being taken from the ordinary more youthful individual.

At times due to the squinted eye of patients which prompts the obscured and fragmented picture. Because of the dryness of the eye additionally endeavors to same inadequacy in picture. These two cases are the aftereffects of Figure 1a. In some circumstance due to the inaccurate and out of focal point of fundus that ought to be precisely lined up with continuance.

Fundus photos used to report the irregularities happens because of the infection influencing in eye for the most part for the patients with diabetes mellitus. Normal fundus screening is essential for them since they are should have diabetic retinopathy which prompts the loss of vision [1]. This can 
likewise be utilized to screen the counter malarial treatment, patients with steady migraine and with diastolic weight. Since it causes swollen in the OD for the cerebral pain and weight influenced patients. This can likewise be utilized for patients with blood vessel hypertension which may prompt cerebravascular mischances like stroke. Every one of these issues is to be significantly thought as this is specifically connected with the lives of valuable individuals.

Fundus photography is fundamentally used to review the inconsistencies like macular degeneration, retinal neoplasm; choroid unsettling influences and so on. By distinguishing the retinal changes and discoveries utilizing fundus photography, a clinician can recognize such irregularities as opposed to taking the blood tests as done in the present period. Rather than taking the blood tests every single time from the patient, the location of progress in the fundus photography demonstrates its seriousness. One of the complexities of diabetes prompts the debilitation of vision and forward towards the visual deficiency is diabetic retinopathy (DR) [2,3] which influences the vessels of blood in light touchy tissues i.e. retina which lie in the rear of the eye. This is the real reason among the vision misfortune individuals having diabetes and cause the visual impairment in the working-age grown-ups [1].

Diabetic macular edema (DME) is the real reason for visual deficiency among the working age aggregate people groups. One out of three diabetic individuals presents indications of DR and one out of ten perseveres from its most fierce and vision-threatening structures. DR can be managed utilizing existing dealings, which are effective if analysed early. Since DR is asymptomatic until the point that late in the illness procedure, normal eye fundus examination is required to investigate any changes in the retina. With the expanding strength of diabetes and the maturing populace, it is likely that, in 2025, 333 million diabetic patients universally will involve retinal examination every year. Considering the insufficient number of ophthalmologists, there is a basic require for computerization in the screening system so as to swathe the substantial diabetic masses while decreasing the clinical seat on retina pros. Computerization can be proficient at two levels: in the first place, in seeing cases with DR, and, second, in reviewing these cases. Certainly, the acknowledgment of the ruthlessness level, amid DR reviewing, endures assist suitable and unswerving suggestion to treatment focuses.

At initially, DR may grounds no signs or scarcely docile vision inconveniences. Accordingly nobody go for customary screening examination at the end of the day, it can attach to visual deficiency. The situation can stretch out in any individual who has type 1 or type 2 diabetes. DR regularly has no early insight signs. Indeed, even DME which can attach to expedient vision misfortune might not have any advice signs for quite a while. By and large, a man with DME is probably going to have contorted vision, making it difficult to do things like read or drive. Now and again, the vision will show signs of improvement or more awful amid the day.

In the main stage, which is called non-proliferative diabetic retinopathy (NPDR) there are no side effects, the signs are not obvious to the eye and patients will have $20 / 20$ vision. The best way to recognize NPDR is by fundus photography, in which smaller scale aneurysms can be seen. In the event that there is diminished vision, fluoresce in angiography should be possible to see the back of the eye. Narrowing or blocked retinal veins can be seen plainly and this is called retinal ischemia.

In the second stage, strange fresh recruits vessels frame at the back of the eye as a major aspect of proliferative diabetic retinopathy; these can blast and obscure the vision, in light of the fact that these fresh recruits' vessels are delicate. The first run through when draining happens, it may not be exceptionally extreme. Much of the time, it will leave only a couple of bits of blood, or spots skimming in a man's visual field; however the spots regularly leave following couple of hours.

These spots are frequently taken after inside a couple of days or weeks by a considerably more prominent spillage of blood, which obscures the vision. In extraordinary cases, a man may just have the capacity to tell light from dull in that eye. It might take the blood anyplace from a couple of days to months or even a long time to clear from within the eye, and now and again the blood won't clear. These kinds of huge hemorrhages have a tendency to happen more than once, regularly amid rest. The objective of our framework is to distinguish both miniaturized aneurysms and hemorrhages in the retinal picture of the diabetic patient and furthermore helps in recognizing the state of the diabetic individual. Our application not just aides in distinguishing the aneurysms and hemorrhages yet additionally help in finding the retinal state of the patient. Figure 2 shows the fundus images with micro aneurysms and hemorrhages.

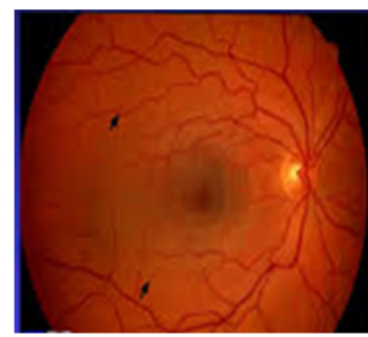

(a)

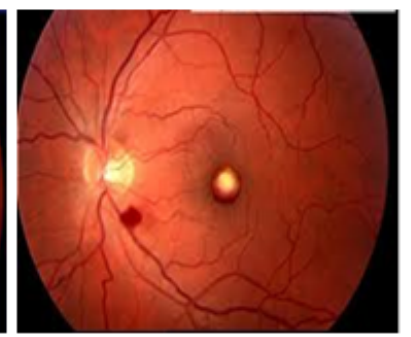

(b)
Figure 2. (a) Micro aneurysms (b) hemorrhages.

A retinal smaller scale aneurysm is a little zone of blood jutting from a conduit or vein in the back of the eye. These projections may open and break blood into the retinal tissue encompassing it. Retinal discharge is seeping from the veins in the retina, inside your eye. Retina is the thin layer that lines the back of your eye.

The exploration centers around the advancement of a programmed telemedicine framework for $\mathrm{PC}$ supported screening and reviewing of DR. Since PC investigation can't supplant the clinician, the framework goes for recognizing fundus pictures with suspected sores and at arranging them by seriousness. At that point, the commented on pictures are sent to a human master for survey, beginning with the suspected 
most serious cases. Such a programmed framework can diminish the authority's weight and examination time, with the extra preferences of objectivity and reproducibility. Besides, it can help to quickly recognize the most serious cases and to concentrate clinical assets on the cases that need more pressing and particular consideration.

The destinations of the venture are to enhance the execution utilizing robotized or semi-computerized divisions, to identify both smaller scale aneurysms and discharge in the retinal picture and it performs well for extensive varieties in light all through a picture. The upsides of the examination incorporate the simple high precision and practical and check time is for the most part less. Section 2 depicts the review of writing records made on such research establishments. Section 3 gives the proposed framework diagram and the square of units expected to play out the procedure. The individual parts expected to deal with the proposed procedure are talked about in detail. Section 4 gives the outcomes and dialog made on the fundus pictures taken from the $\mathrm{ROCH}$ database and the order classes are specified. Section 5 bargains about the conclusion and discusses the improvements to be made on the techniques talked about.

\section{Literature Survey}

In [2], a computerized strategy to find the optic nerve in pictures of the visual fundus. It utilizes a novel calculation called fluffy joining to decide the beginning of the vein arrange. It assesses by utilizing 31 pictures of solid retinas and 50 pictures of infected retinas, containing such different side effects as convoluted vessels, choroidal neovascularisation, and hemorrhages that totally darken the real nerve. Fuzzy logic has been utilized to deal with the idea of incomplete truth, where reality esteem may run between completely true and false.

In [4], the strong location of red sores in advanced shading fundus photos is a basic advance in the improvement of computerized screening frameworks for diabetic retinopathy. Framework were to be utilized as an apparatus to help and HE in distinguishing red sores, execution can be estimated utilizing free-reaction working trademark (FROC) examination. While helping a human, the objective of the framework is identify however many injuries as would be prudent while not producing an exorbitant number of FPs.

In [3], a technique for the programmed location of small scale aneurysms (MAs) in shading retinal pictures is proposed in this paper. The acknowledgment of MAs is a basic advance in the finding and evaluating of diabetic retinopathy. The proposed technique acknowledges MA identification through the investigation of directional cross-area profiles focused on the nearby most extreme pixels of the pre-handled picture. The proposed cross-segment investigation based element extraction and highlights could be abused in other therapeutic picture handling related undertakings, particularly in instances of unusual situations.

The current framework have a few nearby order issues that prompts misclassification issues that may emerge while consolidating earlier information about the state of the coveted structures on the learning procedure [5-23]. CRFs can give such data through the match astute possibilities. All the current techniques that consider the data which is nearby to every pixel through picture channels, overlooking valuable data from shapes and structures show in the picture. This strategy did not perform well for substantial varieties in lighting all through a picture, yet this happened for just a single picture out of the 40 tried from the two databases. It is conceivable to utilize just the skeleton of the divisions for the extraction of shape.

\section{Proposed System}

Figure 3 demonstrates the square outline of the proposed framework which is a six stage process that begins its task from spatial adjustment and the preprocessing of the info pictures are expected to improve the information pictures taken for activity. The OD from the info picture ought to be expelled as our emphasis is basically on alternate highlights bolstered in the fundus picture. At that point the arrangement is combined with RF and Principal Component Analysis (PCA) that includes a double method to affirm the innovation of the fundus picture whether it gets influenced or not.

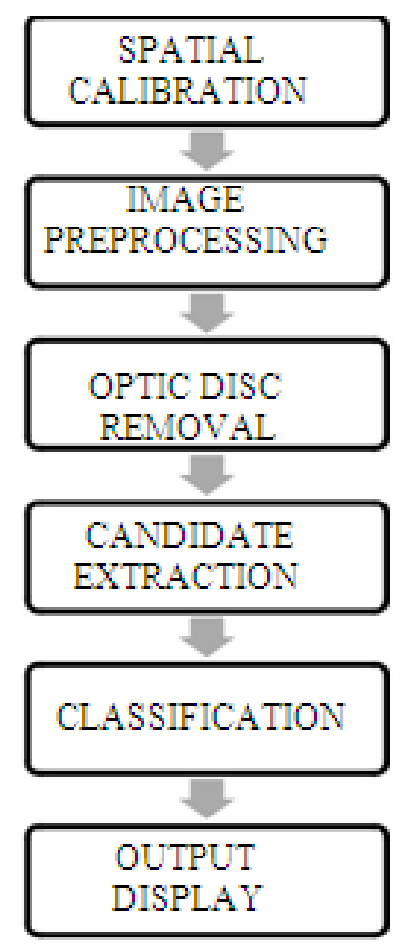

Figure 3. Block diagram of proposed methodology.

Location of the two MAs and HEs in eye sore Images are finished by the proposed technique and the examination, for example, estimations of distances across and tortuosity of the vessels, order of veins and conduits, count of the blood vessel venous proportion are additionally made. The automated or semi-robotized division strategies would have changes in productivity and exactness. The complete study is made on the pictures of Retinopathy Online Challenge (ROCh) database [23]. This public database comprises 50 images for training 
and 50 images for testing with pixel size of $720 \times 720$ which are all JPEG images that causes a ton to distinguish the defencelessness and happens to satisfy the goal of the article to differentiate the normal and abnormal fundus images.

Figure 4 portrays the design to be taken after for demonstrating the right arrangement comes about. By following this sort of system early recognition of the variations from the norm made without the loss of the blood tests every last time that happens while checking for the irregularity.

\section{Spatial calibration}

Spatial calibration is utilized to adjust the distinctive picture resolutions where the pictures are not resized but rather the breadth of the Region of Interest (ROI) is a size invariant one that must be considered. This is sensible since the greater part of the pictures for DR screening are procured with a field of view (FOV) of $45^{\circ}$ which includes concealing the info picture.

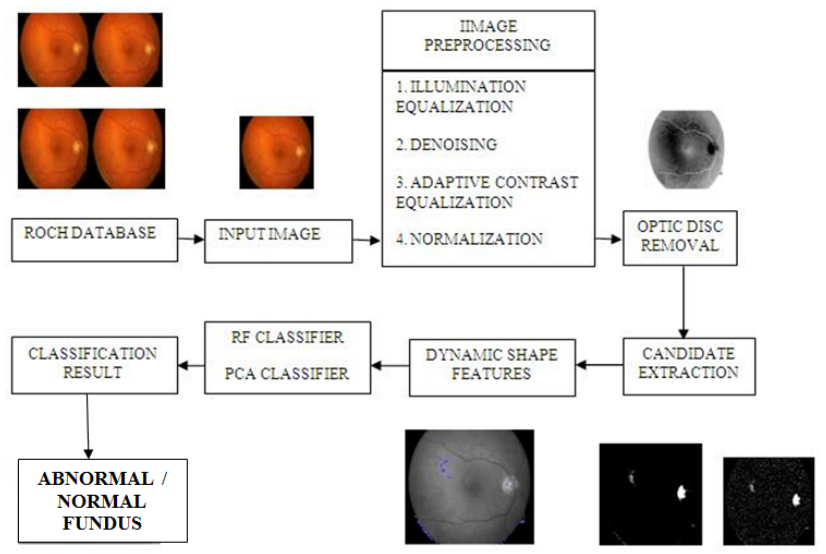

Figure 4. Architecture diagram of proposed methodology.

Figure 5 shows the masked input done by the spatial calibration steps done on the input images. Figure 6 depicts the way how the spatial calibration operation can be executed.

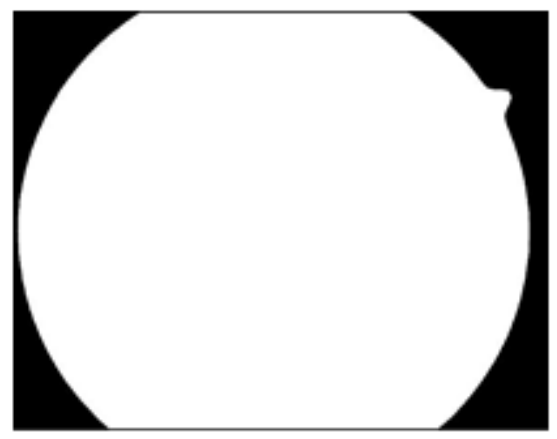

Figure 5. Masked input by spatial calibration.

\section{Image preprocessing}

The enlightenment of the retina is frequently non-uniform, prompting neighborhood glow and differentiation variety. Injuries might be not really unmistakable in territories of poor difference as well as low shine. In addition, in a telemedicine setting, pictures are variable as far as shading and quality. Thus, pre-preparing steps are required to address these issues. Figure 7 demonstrates the outline of all the picture preprocessing steps.

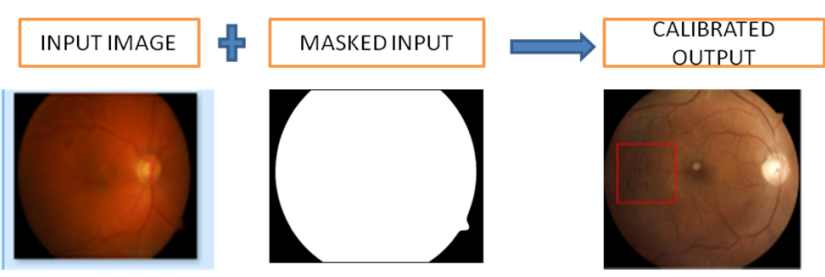

Figure 6. Spatial calibration steps.

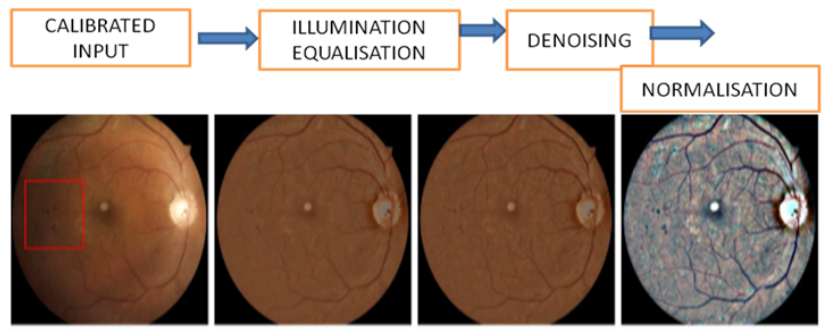

Figure 7. Image preprocessing steps.

\section{Illumination equalization}

To conquer the vignette impact enlightenment evening out technique is utilized. A vast mean channel of breadth is connected to each shading segment of the first picture keeping in mind the end goal to appraise its enlightenment. At that point, the subsequent shading picture is subtracted from the first one to remedy for potential shade varieties. At long last, the normal power of the first channel is added to keep a similar shading range as in the first picture. The illumination equalization are done in the images to make the whole image more uniform, because the non-uniformity may be due to the reflection and attenuation of illumination light in the different eye layers areas along the various illumination paths. Figure 8 shows the output of illumination equalization.

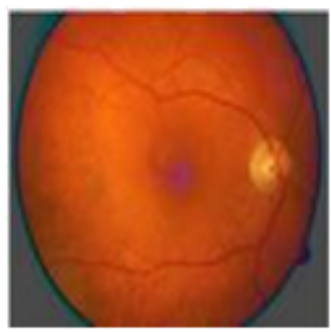

Figure 8. Illumination equalization.

\section{Denoising}

A little mean filter of distance across is connected to each shading channel of the subsequent picture keeping in mind the end goal to constrict the clamor coming about because of the securing and pressure ventures without smoothing the injuries. The objective of denoising is that the image corrupted by noise 
lacks its originality factor. To retain the effectiveness of the original images, this process initiated. Figure 9 focuses on expelling the clamor from the information pictures.

\section{Adaptive contrast equalization}

The complexity float is approximated utilizing the nearby standard deviation processed for every pixel in an area of distance across, for each shading channel. Regions with low standard deviation demonstrate either low difference or smooth foundation. To upgrade low complexity territories, we hone the subtle elements in the particular areas utilizing for each shading channel independently is done by the adaptive contrast equalization. Figure 10 gives the levelling in the difference made on the info picked picture.

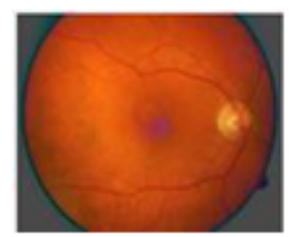

Figure 9. Denoised image.

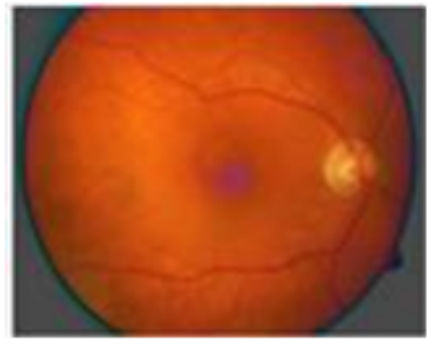

Figure 10. Adaptive contrast equalization.

\section{Color normalization}

Color or shading standardization is fundamental keeping in mind the end goal to acquire pictures with an institutionalized shading range. We perform, in each shading channel of, histogram extending and cutting in the range, where and are the mean and standard deviation of the shading divert in the ROI. The aftereffects of histogram evening out have a tendency to have an overstated blue channel and look unnatural, because of the way that in many pictures the conveyance of the pixel esteems is normally more like a Gaussian circulation, as opposed to uniform. Color normalization allows for object recognition techniques based on color to compensate for these variations. Figure 11 demonstrates the consequence of shading standardized picture.

\section{Optic disc removal}

Beginning from the pre-handled picture, we first utilize an entropy-based way to deal with assess the area of the OD's middle. Essentially, the OD is situated in a high force locale where the vessels have maximal directional entropy. An ensuing advancement step at that point gauges the OD's sweep and refines its position. This comprises in convolving a multiscale ring-formed coordinated channel to the picture in a subROI focused on the principal estimation of the OD's inside, of span equivalent to $33 \%$ of the ROI's sweep. Figure 12a demonstrates the OD evacuated picture and the Figure $12 \mathrm{~b}$ demonstrates the reasonable and prepared OD expelled fundus picture.

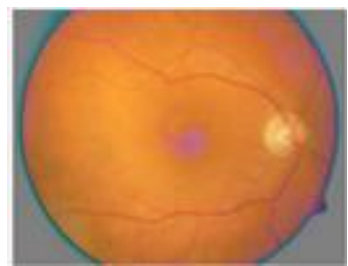

Figure 11. Color normalized input image.

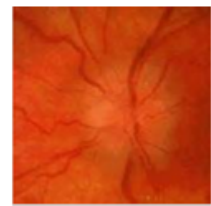

(a)

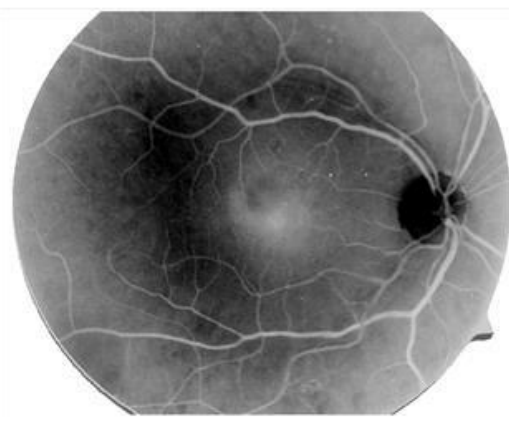

(b)
Figure 12. (a) Optic disc removal (b) Clear and processed OD removed image.
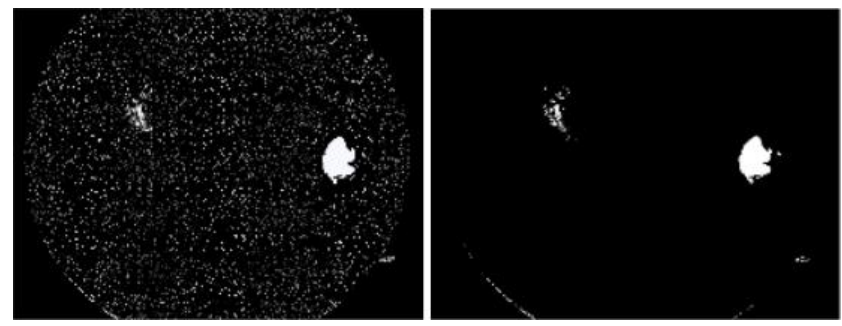

Figure 13. Candidate extraction.
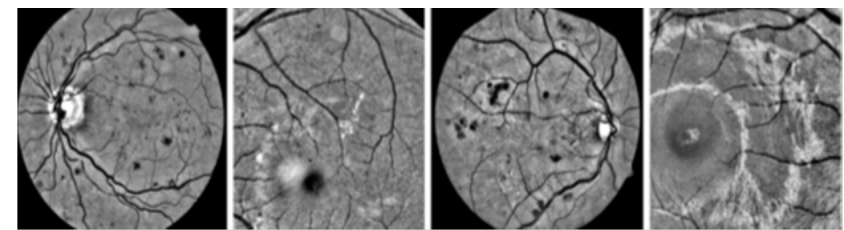

Figure 14. Candidate extraction procedure.

\section{Candidate extraction}

In the green channel, MAs and HEs show up as structures with neighborhood negligible force. A beast compel approach is separate all the provincial minima. A local least is a gathering of associated pixels of steady power, to such an extent that all the neighboring pixels have entirely higher forces. Lamentably, this strategy is very touchy to commotion. Contingent upon the 
smoothness of the picture, the quantity of territorial minima would thus be able to be substantial. Difference and enlightenment evening out is imperative now. Without these pre-handling steps, worldwide difference and power thresholding would be hard to accomplish. Moreover, all hopefuls whose separation to the OD's inside is littler than the OD's span are expelled from the arrangement of competitors and not considered any further [4,6]. Figure 13 demonstrates the highlights or the competitors separated from the given picture and Figure 14 delineates the hopeful extraction process.

\section{Dynamic shape feature detection}

Among the candidates, a few areas relate to non-sores, for example, vessel sections and remaining clamor in the retinal foundation. To separate between these false positives and genuine sores, a unique arrangement of highlights, the DSFs, primarily in view of shape data, is proposed. At each flooding level, for every hopeful, six shape qualities are registered on the catchment bowl. They are

1. Relative area: Number of pixels in, partitioned by the aggregate number of pixels in the ROI.

2. Elongation: With and the width and length, separately, of the bouncing box of arranged along its real hub.

3. Eccentricity: With and the width and length, individually, of the jumping box of arranged along its significant pivot.

4. Circularity: Ratio of the region over its squared border and increased.

5. Rectangularity: Ratio of the territory of over the region of its bouncing box situated along its real hub.

6. Solidity: Ratio of the zone of over the region of its arched frame.

Figure 15 demonstrates the shape includes that are distinguished from the given picture that are appeared as blue dabs.

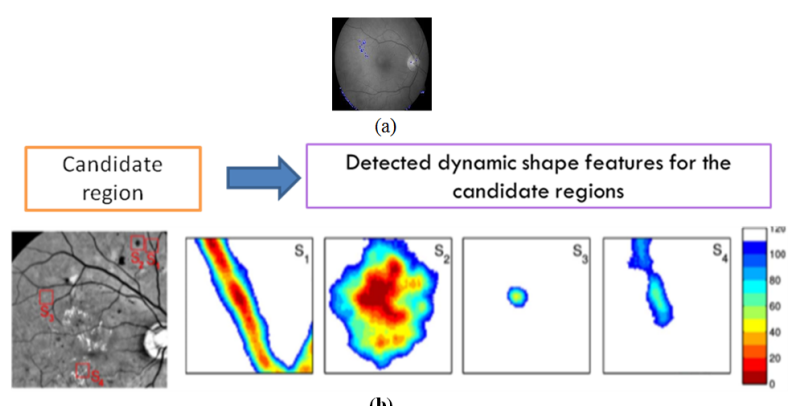

(b)

Figure 15. (a) Features detected from the image (b) Dynamic shape feature detection process.

\section{Classification}

RF classifier: To distinguish between lesions and non-lesions, a Random Forest (RF) classifier. This ground-breaking approach has been broadly utilized as a part of $\mathrm{PC}$ vision in the course of the most recent couple of years, because of its various points of interest. It is helpful for non-straight order with high-dimensional and uproarious information. It is hearty against anomalies and over-fitting. Additionally, it joins a verifiable highlights determination step. A RF is a mix of choice trees prepared freely utilizing bootstrap tests drawn with substitution from the preparation set. Every hub is part utilizing the best of a haphazardly chose subset of highlights picked, as indicated by the lessening in the Gini record. The RF returns, for every applicant, a likelihood of being an injury, equivalent to the extent of trees restoring a positive reaction. Figure 16 shows the classified output of the given findus image.

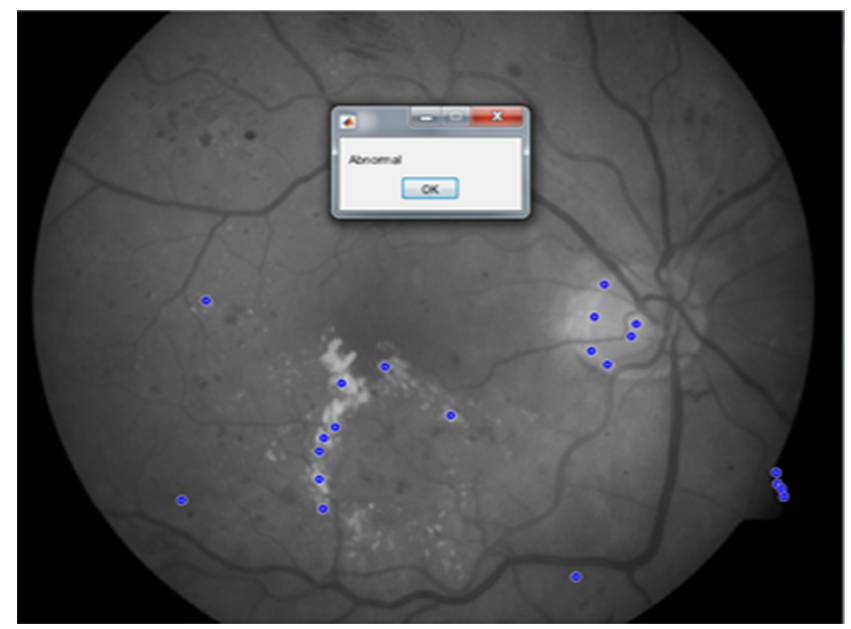

Figure 16. Classification result.

\section{Principal component analysis (PCA) classifier}

Principal component analysis is a technique for distinguishing fewer uncorrelated factors, called "key segments", from an extensive arrangement of information. The objective of chief parts examination is to clarify the most extreme measure of difference with the least number of primary segments. Essential parts investigation is usually utilized as a part of the sociologies, statistical surveying, and different ventures that utilization vast informational index.

Primary parts examination is normally utilized as one stage in a progression of investigation. It is utilized to decrease the quantity of factors and to maintain a strategic distance from multi co-linearity, or when excessively numerous indicators in respect to the quantity of perceptions. The highlights acquired are ordered utilizing key part examination in light of the segment arrangement, group and inquiry strategies.

The current philosophies had numerous hindrances and all these can be overwhelmed by the proposed framework classifier that recognizes the injuries and non-sores Random Forest (RF) classifier is the great approach and helpful for nonstraight order with high-dimensional and boisterous information. It is powerful against exceptions and over-fitting. Also, it fuses a certain highlights choice advance. A RF is a blend of choice trees prepared autonomously utilizing bootstrap tests drawn with substitution from the preparation set. Every hub is part utilizing the best of a haphazardly chose subset of highlights picked, as per the diminishing in the Gini 
file. The RF returns, for every applicant, a likelihood of being an injury, equivalent to the extent of trees restoring a positive reaction.
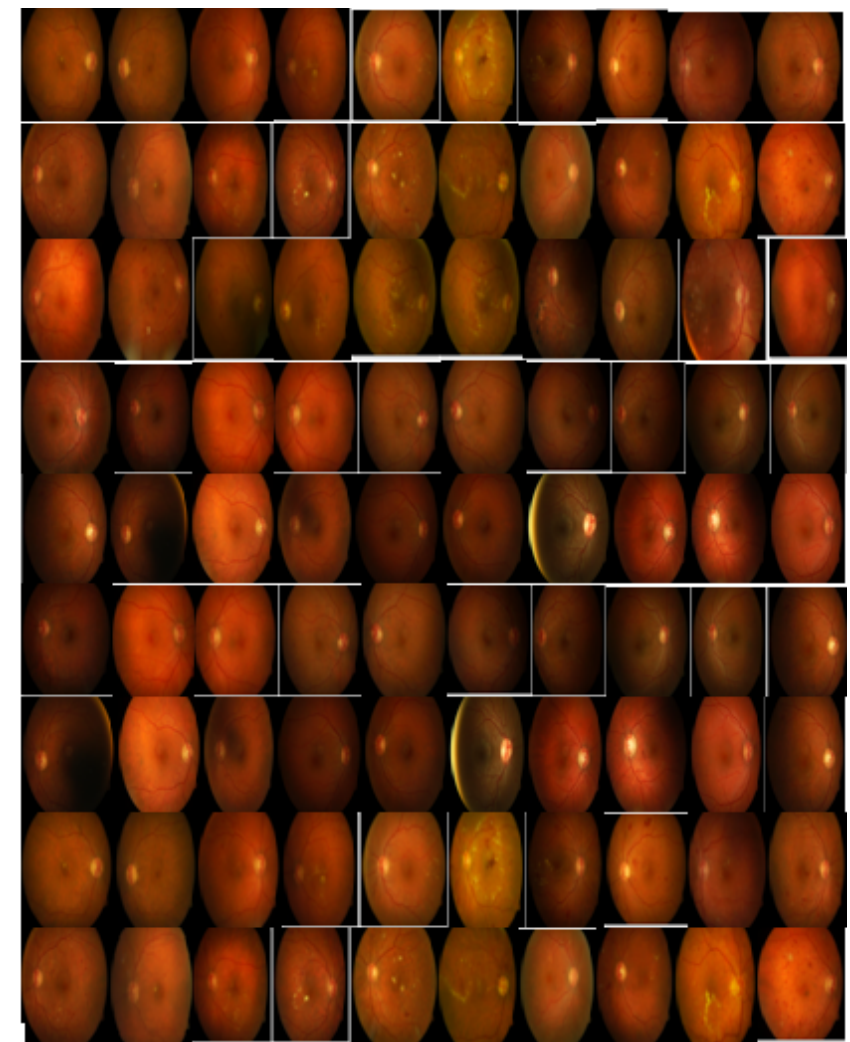

Figure 17. ROCh database.
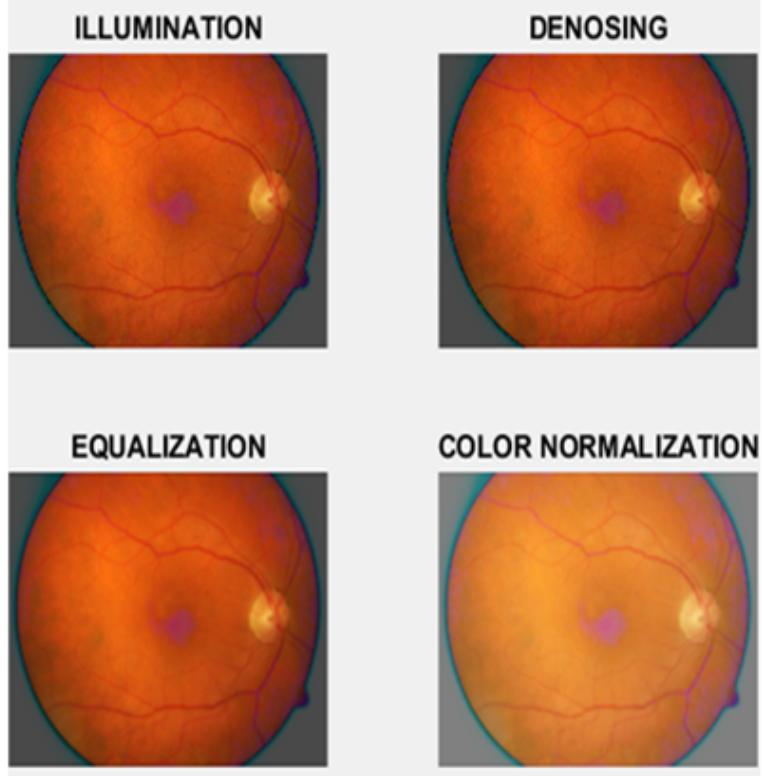

Figure 18. Preprocessing input image.

\section{Results and Discussion}

The experimentation is carried on ROCh database which is shown in Figure 17. This public database comprises 50 images for training and 50 images for testing with pixel size of $720 \times$ 720 which are all JPEG images. The images are shown below are spatial calibrated and do the image preprocessing steps.

Figure 18 shows the pre-processed images of all the images in the database and list down the preprocessing time taken. Figure 19 shows the gray scale converted Image. Figure 20 is the optic disc removed image whereas the Figure 21 is the candidate extracted image and Figure 22 shows the features obtained from dynamic shape and Figure 23 depicts the classification result extracted image.

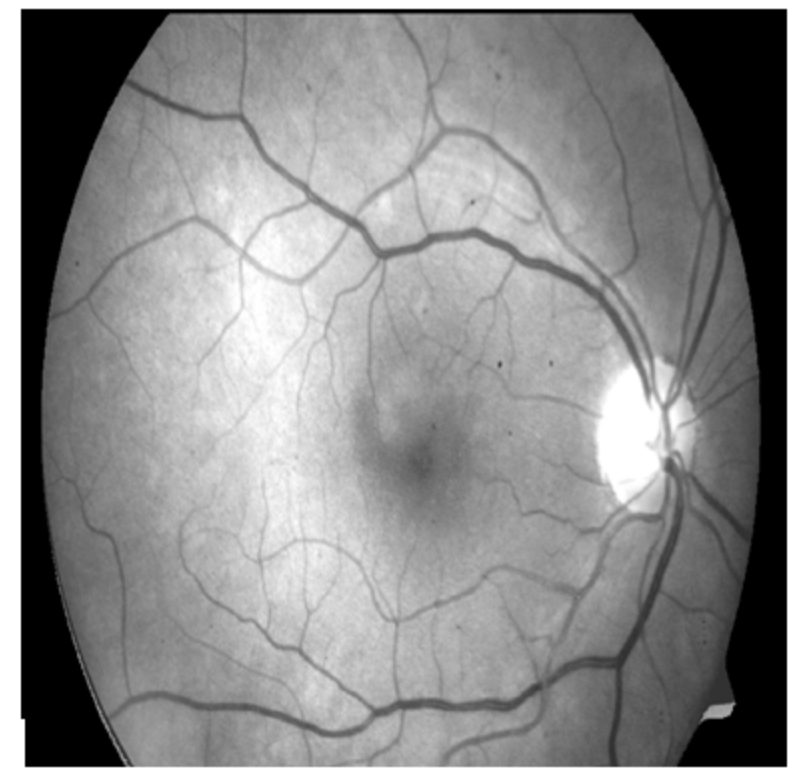

Figure 19. Gray scale converted image.

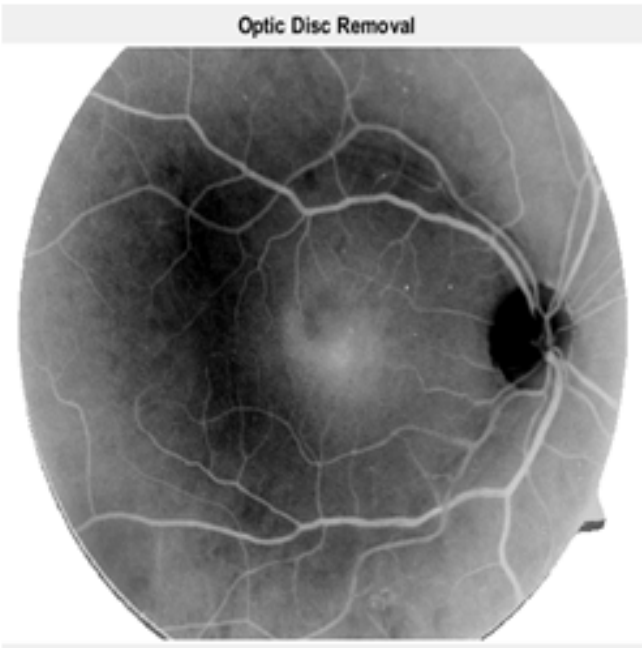

Figure 20. OD removed image.

\section{Classify procedure of classification}

For classification the linear regression and multilayer perceptron functions are used. A graph can be plotted for mean square error along with the time taken for the identified six 
features for an abnormal image is represented as follows in Table 1.

Table 1. Classify data set.

\begin{tabular}{|c|c|c|c|c|c|c|}
\hline \multirow{2}{*}{ Feature } & \multicolumn{2}{|l|}{ Linear regression } & \multicolumn{2}{|l|}{ Multilayer perceptron } & \multicolumn{2}{|l|}{ RBF network } \\
\hline & Mean absolute error & Time taken (s) & Mean absolute error & Time taken (s) & Mean absolute error & Time taken (s) \\
\hline Circularity & 0.0454 & 0.03 & 0.008 & 0.003 & 0.0454 & 0.02 \\
\hline Eccentricity & 0 & 0.02 & 0.0022 & 0.39 & 0.0864 & 0.003 \\
\hline Elongitivity & 0 & 0 & 0.6052 & 0.42 & 0.2365 & 0.02 \\
\hline Solidity & 0 & 0.02 & 0.0036 & 0.52 & 0.767 & 0.52 \\
\hline Relative area & 0 & 0 & 0 & 0.41 & 0.0002 & 0.02 \\
\hline Rectangularity & 0.0004 & 0 & 52.6579 & 0.45 & 540.2727 & 0 \\
\hline
\end{tabular}

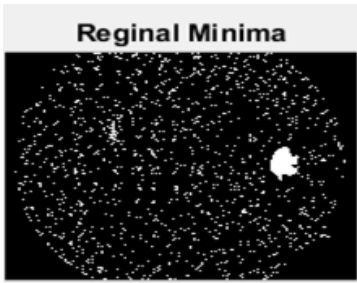

Figure 21. Candidate extracted image.

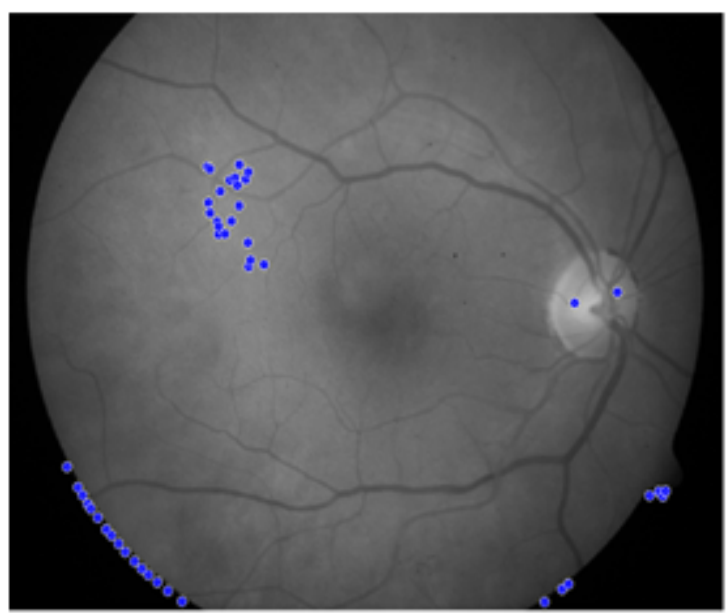

Figure 22. Dynamic shape features.

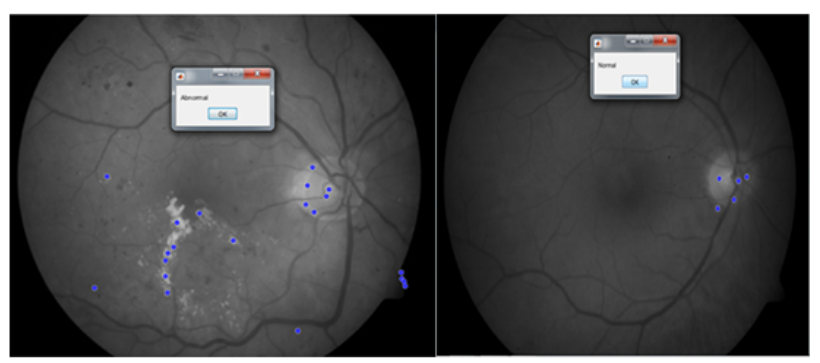

Figure 23. Classification result extracted image.

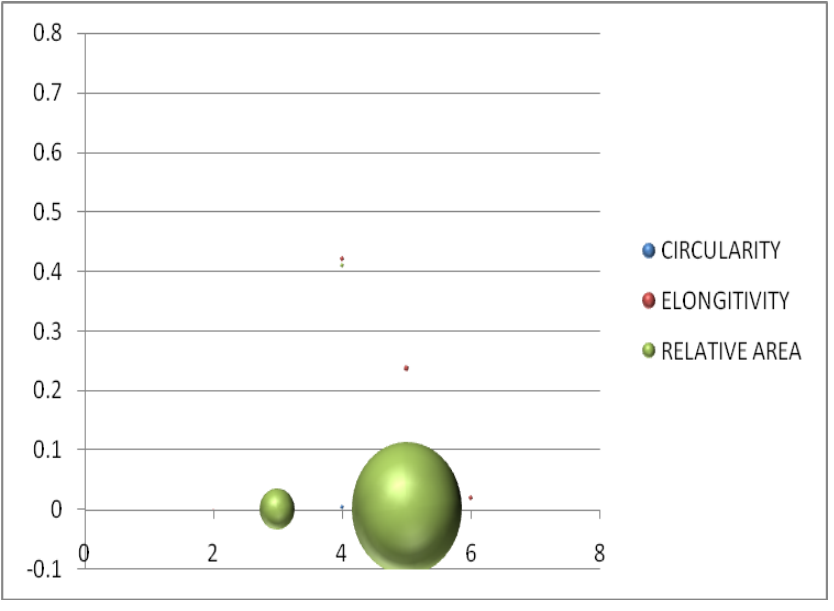

Figure 24. Classification of mean absolute error (Linear regression).

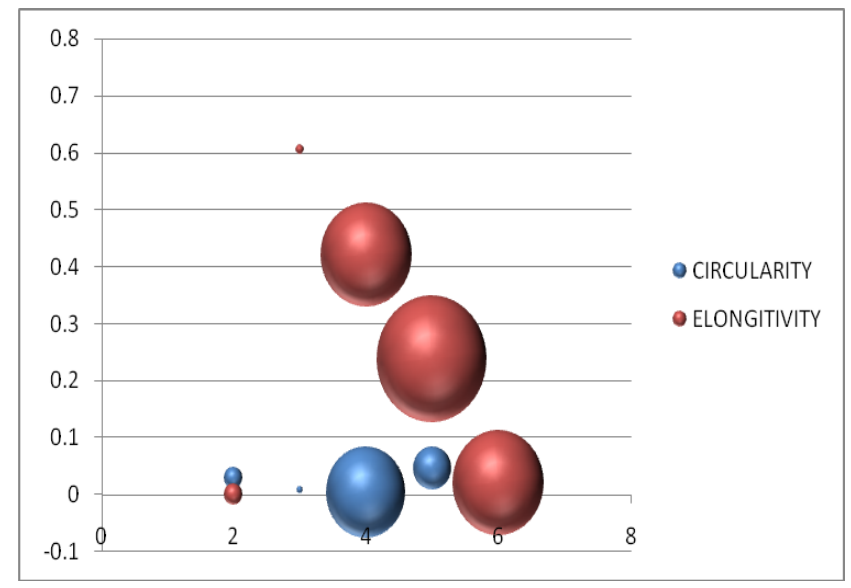

Figure 25. Classification of mean absolute error (Multilayer perceptron).

Similarly the root mean square error for the abnormal image can be plotted against the time can be shown as in Figures 24 and 25. Figure 26 depicts the output of classification using root mean square error. 


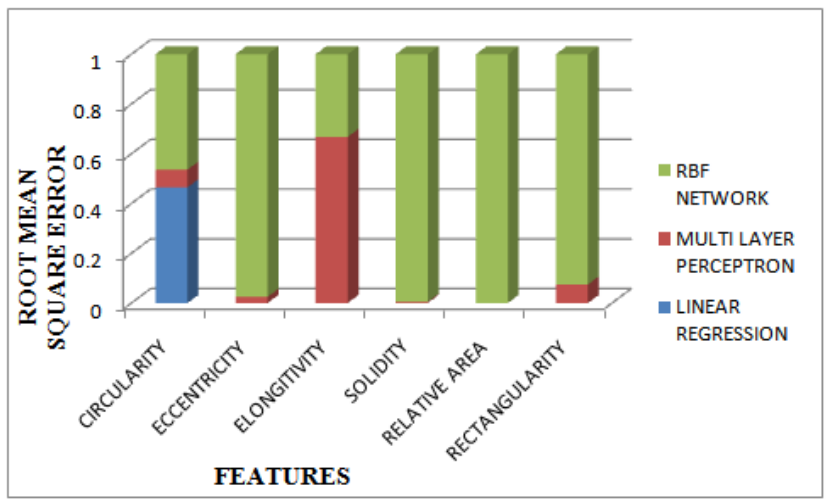

Figure 26. Classification using root mean square error.

\section{Clustering procedure of classification}

An expectation-maximization (EM) calculation is an iterative strategy to discover most extreme probability or maximum a posteriori (MAP) appraisals of parameters in factual models, where the model relies upon surreptitiously dormant factors.
The EM cycle interchanges between playing out a expectation (E) step, which makes a capacity for the desire for the logprobability assessed utilizing the present gauge for the parameters, and an maximization (M) step, which figures parameters expanding the normal log-probability found on the E step. These parameter-gauges are then used to decide the dispersion of the dormant factors in the following E step.

Hierarchical clustering or hierarchical cluster analysis or HCA is a technique for group examination which looks to manufacture a pecking order of groups. Systems for various levelled grouping by and large fall into two sorts:

Agglomerative: This is a "base up" approach: every perception begins in its own bunch, and combines of groups are converged as one climbs the progression.

Divisive: This is a "best down" approach: all perceptions begin in one bunch, and parts are performed recursively as one move down the pecking order. Table 2 shows the clustering data set using EM algorithm and Hierarchical cluster (Figure 27).

Table 2. Clustering data set.

\begin{tabular}{|c|c|c|c|c|}
\hline \multirow{2}{*}{ Feature } & \multicolumn{2}{|l|}{ EM algorithm } & \multicolumn{2}{|l|}{ Hierarchial cluster } \\
\hline & Number of cluster & Time taken (s) & Number of cluster & Time taken (s) \\
\hline Circularity & 1 & 0.08 & 2 & 0 \\
\hline Eccentricity & 1 & 0.28 & 2 & 0 \\
\hline Elongitivity & 1 & 0.14 & 2 & 0.02 \\
\hline Solidity & 1 & 0.36 & 2 & 0 \\
\hline Relative area & 1 & 0.14 & 2 & 0 \\
\hline Rectangularity & 1 & 0.14 & 2 & 0 \\
\hline
\end{tabular}

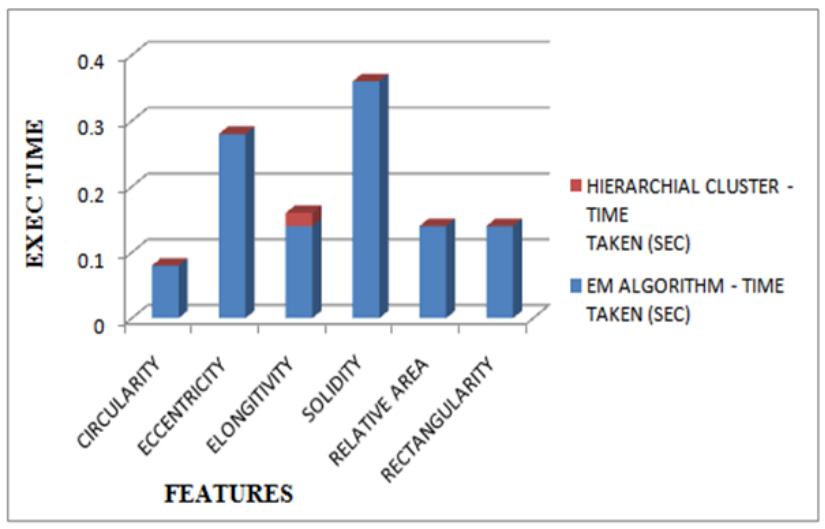

Figure 27. Clustering of data set-Time taken.

\section{Conclusion and Future Enhancement}

A novel red injury location strategy in light of another arrangement of shape includes, the DSFs, was displayed and assessed on six unique databases. The outcomes show the solid execution of the proposed strategy in recognizing the two MAs and HEs in fundus pictures of various determination and quality and from various securing frameworks. The strategy outflanks numerous cutting edge approaches at both per-injury and per-picture levels. DSFs have turned out to be strong highlights, profoundly fit for separating amongst sores and vessel sections. The idea of DSFs could be misused in different applications, especially when the articles to be recognized don't indicate clear limits and are hard to fragment exactly. Additionally work concentrating on splendid injury and neo vessel location will finish the proposed framework and permit programmed DR evaluating.

Additionally work concentrating on brilliant injury and neo vessel recognition will finish the proposed framework and permit programmed DR reviewing. OD step is connected and the proposed technique ended up being ready to recognize vessel bifurcations and intersections from MAs rather well, in any case, MA-like structures do show up on the optic plate, and since the differentiation is high in this district, some of the time a somewhat high score is appointed. In spite of the fact that the proposed technique indicated persuading execution, this could most likely be additionally enhanced by barring MA 
identifications inside this locale and the proposed framework examination in light of highlight extraction and list of capabilities could be abused in other restorative picture preparing related assignments, particularly in instances of variation from the norm discovery that include the acknowledgment of almost round or marginally stretched structures in a picture. The cotton fleece spots [14] in DR pictures are not gathered in the proposed strategy. This can be upgraded later on improvement.

\section{References}

1. Carlos HM, Xenophon Z, Areti T, Panagiota A, Stella D, Antonis AA. FIRE: Fundus Image Registration Dataset. J Model Ophthalmol 2017; 4: 16-28.

2. Hoover A, Goldbaum M. Locating the optic nerve in a retinal image using the fuzzy convergence of the blood vessels. IEEE Trans Med Imag 2003; 22: 951-958.

3. Lazar I, Hajdu A. Retinal microaneurysm detection through local rotating cross-section profile analysis. IEEE Trans Med Imag 2013; 32: 400-407.

4. Niemeijer M, van Ginneken B, Staal J, Suttorp-Schulten MS, Abràmoff MD. Automatic detection of red lesions in digital color fundus photographs. IEEE Trans Med Imaging 2005; 24: 584-592.

5. Quellec G, Lamard M, Josselin PM, Cazuguel G, Cochener B, Roux C. Optimal wavelet transform for the detection of microaneurysms in retina photographs. IEEE Trans Med Imaging 2008; 27: 1230-1241.

6. Seoud L. Automatic detection of microaneurysms and haemorrhages in fundus images using dynamic shape features. Proc IEEE 11th Int Symp Biomed Imag Beijing 2014; 101-104.

7. Baudoin C, Lay B, Klein J. Automatic detection of microaneurysms in diabetic fluorescein angiographies. Revue Dépidémiologie et de Santé Publique 1984; 32: 254-261.

8. Cheung N, Mitchell P, Wong TY. Diabetic retinopathy. Lancet 2010; 376: 124-136.

9. Cree M, Olson J, McHardy K, Sharp P, Forrester J. A fully automated comparative micro aneurysm digital detection system. Eye 1997; 11: 622-628.

10. Ding J, Wong TY. Current epidemiology of diabetic retinopathy and diabetic macular edema. Curr Diabetes Rep 2012; 12: 346-354.

11. Frame AJ. A comparison of computer based classification methods applied to the detection of microaneurysms in ophthalmic fluorescein angiograms. Comput Biol Med 1998; 28: 225-238.

12. Gan D. Diabetes atlas (2nd Edn.). Brussels Int 2003.

13. Mizutani A, Muramatsu C, Hatanaka Y, Suemori S, Hara T, Fujita H. Automated microaneurysm detection method based on doublering filter in retinal fundus images. SPIE Med Imag Comp Aid Diagn 2009; 7260: $72601 \mathrm{~N}-72601 \mathrm{~N}-8$.

14. Ravishankar SJ, Mittal A. Automated feature extraction for early detection of diabetic retinopathy in fundus images. Proc IEEE Conf Comput Vis Pattern Recogn 2009; 210-217.

15. Sinthanayothin C. Automated detection of diabetic retinopathy on digital fundus images. Diabetic Med A J Brit Diabetic Assoc 2002; 19: 105-112.

16. Spencer T, Phillips RP, Sharp PF, Forrester JV. Automated detection and quantification of micro aneurysm in fluorescein angiograms. Graefes Arc Clin Exp Ophtalmol 1992; 230: 36-41

17. Walter T. Automatic detection of micro aneurysms in color fundus images. Med Image Anal 2007; 11: 555-566.

18. Wilkinson CP. Proposed international clinical diabetic retinopathy and diabetic macular edema disease severity scales. Ophthalmology 2003; 110: 1677-1682.

19. Yau JW, Rogers SL, Kawasaki R, Lamoureux EL, Kowalski JW, Bek T, Chen SJ, Dekker JM, Fletcher A, Grauslund J, Haffner S, Hamman RF, Ikram MK, Kayama T, Klein BE, Klein R, Krishnaiah S, Mayurasakorn K, OHare JP, Orchard TJ, Porta M, Rema M, Roy MS, Sharma T, Shaw J, Taylor H, Tielsch JM, Varma R, Wang JJ, Wang N, West S, Xu L, Yasuda M, Zhang X, Mitchell P, Wong TY; Meta-Analysis for Eye Disease (METAEYE) Study Group. Global prevalence and major risk factors of diabetic retinopathy. Diabetes Care 2012; 35: 556-564.

20. Zhang $X$. Exudate detection in color retinal images for mass screening of diabetic retinopathy. Med Image Anal 2014; 18: 1026-1043.

21. Zhang B, Wu X, You J, Li Q, Karray F. Detection of micro aneurysms using multi-scale correlation coefficients. Patt Recogn 2010; 43: 2237-2248.

22. Shruti G, Rida A. A review paper on detection of glaucoma using retinal fundus images. Int J Res Appl Sci Eng Technol 2016; 4.

23. Niemeijer M. Retinopathy online challenge: automatic detection of microaneurysms in digital color fundus photographs. IEEE Trans Med Imaging 2010; 29: 185-195.

\section{*Correspondence to}

Beulah David D

Department of Computer Science and Engineering

Sathyabama University

India 